\title{
Theory of Stagnation-Point Spray Flame Ignition
}

\author{
G. Kats ${ }^{1}$, J.B. Greenberg ${ }^{* 1}$ \\ ${ }^{1}$ Aerospace Engineering, Technion, Israel Institute of Technology, Haifa, Israel \\ *Corresponding author: aer9801@technion.ac.il
}

\begin{abstract}
A theory of stagnation-point spray flame ignition by an isothermal hot surface is presented for the first time. A mixture of fuel droplets and air flowing against an isothermal hot surface (such as a hot ignition probe) is considered. The spray of droplets is modelled using the sectional approach and a mono-sectional case is adopted for simplicity. A single global chemical reaction is assumed for the case when ignition occurs. The mathematical analysis makes use of a small parameter that is exploited for an asymptotic approach. The analysis produces a criterion for ignition that includes effects of the flow field, the reactants and the fuel spray-related parameters. Numerical computations reveal the way in which the latter impact on whether ignition will occur or not.
\end{abstract}

\section{Keywords}

spray flame; ignition; stagnation-point flow

\section{Introduction}

The theory of spray flame ignition has received rather sparse attention in the literature. The ignition process is generally classified by two possibilities: (a) auto-ignition - which is caused by chain branching or thermal feedback in homogeneous/heterogeneous mixtures without exposure to an external heat source, or (b) forced ignition which involves an external source of energy, e.g. an electrical spark, a heated surface, a shock wave, a pilot flame etc. The most common type of forced ignition is a spark generated by a breakdown voltage between two electrodes. The reason for spark ignition popularity in aerospace systems is the aircraft platform requirement to place the ignition system in a constant location and to ignite the cold mixture within a specific time [1]. In the current work we consider forced ignition of a spray of liquid fuel droplets in an oxidising environment.

The problem of forced ignition in gaseous or two phase (gas-liquid) mixtures can be analysed using two quite different mathematical approaches. For analysis of single droplet ignition it was pointed out [2] that either a quasisteady state analysis can be adopted, in which conditions for the existence of a steady state solution are sought as being indicative of the occurrence of ignition, or a full time-dependent description of the physics can be employed to determine if evolution to a successful or failed state is achieved. These two different approaches were previously used in the context of laminar gaseous diffusion flames for examining conditions for flame extinction [3-5]. Interestingly, it was demonstrated in [3] that the so-called static condition for flame extinction did not necessarily coincide with the dynamic condition. Nevertheless, the static or quasi-steady state approach does provide useful information concerning those factors that play a role in extinction/ignition.

For spray ignition the dynamic approach generally requires numerical simulations [6-8]. Recently, a combination of analytical/numerical tools was utilized to tackle the time-dependent approach to spray flame ignition $[9,10]$.

In $[1,11]$ a full three-dimensional DNS study of turbulent mono-size spray flame spark ignition was carried out using a detailed chemical kinetic scheme. This enabled the different scenarios resulting from use of either dispersed or dense sprays to be studied comprehensively. Such DNS studies provide valuable information but are computationally intensive and, as such, have limitations.

In the current paper we adopt a static/steady-state approach for studying the problem of laminar spray flame ignition at the stagnation point of an isothermal hot surface. This configuration, for gas flames, was investigated by Law [12], Chen et al. [13] and Hsu and Lin [14]. It is a useful paradigm, from both fundamental and practical perspectives. In terms of the theory, a similarity solution is admitted which enables the two-dimensional configuration to be reduced to a one-dimensional form thereby facilitating insight into the basic physics/chemistry. In practical terms, such configurations appear in chemically reacting flows such as the hot tip of a projectile or a heated ignition probe. Furthermore, the influence of stretch can be readily controlled and studied in such a set-up $[13,14]$.

Here we consider for the first time the ignition of a fuel spray-air mixture in stagnation-point flow. We derive a new analytical condition for ignition of the mixture and examine the role of the spray in determining whether ignition is achieved or not. 


\section{Mathematical Model}

The configuration we consider is shown in Fig. 1. A steady laminar flow of a constant density fluid, comprised of fuel vapor, fuel droplets, oxygen and a diluent, approaches the stagnation point $\eta=0$, where $\eta$ is a similarity variable to be described later. A hot spot is generated at the stagnation point due to a prescribed temperature $T_{W}$. Under appropriate conditions the consequent thermal field will enable the formation of steady state premixed spray flame. At the current stage we assume a global, irreversible, one step reaction described by Arrhenius's law can be employed: fuel + oxidant $\rightarrow$ products + heat. In the context of flame ignition a more detailed chemical mechanism is probably more accurate but the one-step mechanism we use provides a reasonable starting point if the role of the spray on ignition is the main focus of attention.

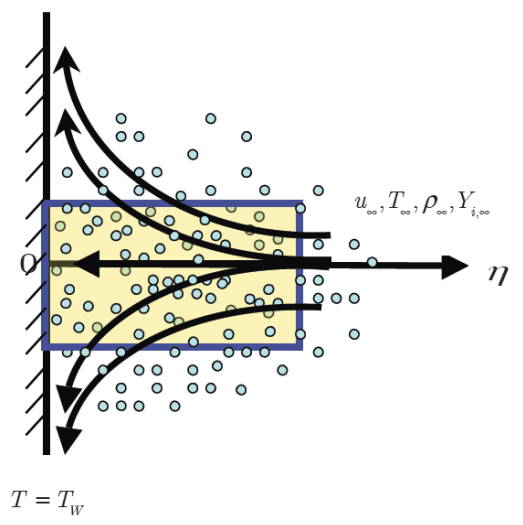

Figure 1: Configuration for stagnation-point ignition by an isothermal hot surface of a premixed fuel spray oxidant mixture.

In Cartesian coordinates the governing equations are the conventional boundary-layer-type conservation equations [15], supplemented by a conservation equation relating to the liquid fuel in the spray.

Continuity:

$\frac{\partial(\rho u)}{\partial x}+\frac{\partial(\rho v)}{\partial y}=0$

Momentum:

$\rho u \frac{\partial u}{\partial x}+\rho v \frac{\partial u}{\partial y}-\frac{\partial}{\partial y}\left(\mu \frac{\partial u}{\partial y}\right)=\rho_{\infty} u_{\infty} \frac{d u_{\infty}}{d x}$

Energy:

$\rho u \frac{\partial T}{\partial x}+\rho v \frac{\partial T}{\partial y}-\frac{\partial}{\partial y}\left(\frac{\lambda}{c_{p}} \frac{\partial T}{\partial y}\right)=\omega-L S_{v}$

Fuel vapor:

$\rho u \frac{\partial Y_{F}}{\partial x}+\rho v \frac{\partial Y_{F}}{\partial y}-\frac{\partial}{\partial y}\left(\rho D_{F} \frac{\partial Y_{F}}{\partial y}\right)=-\omega+S_{v}$

Oxygen:

$\rho u \frac{\partial Y_{O}}{\partial x}+\rho v \frac{\partial Y_{O}}{\partial y}-\frac{\partial}{\partial y}\left(\rho D_{O} \frac{\partial Y_{O}}{\partial y}\right)=-\omega$

Liquid Fuel:

$$
\rho u \frac{\partial Y_{d}}{\partial x}+\rho v \frac{\partial Y_{d}}{\partial y}=-S_{v}
$$

where the chemical source term is

$\omega=\rho \frac{A}{a} Y_{F} Y_{O} \exp \left(-\frac{E}{R T}\right)$

and the vaporization term is

$S_{v}=\rho C Y_{d} H\left(T-T_{v}\right)$ 
In these equations $u, v$ are the velocity components in the $x, y$ directions, respectively, $\rho$ is the density, $T$ is the temperature, $\lambda$ the thermal conductivity, $c_{p}$ the specific heat, $L$ the latent heat of vaporization of the liquid fuel, $Y_{F}, Y_{O}, Y_{d}$ are the mass fractions of the fuel vapor, oxygen and liquid fuel, respectively, $a$ is the strain rate, $A$ the pre-exponential constant, $E$ the activation energy, $R$ the universal gas constant, $C$ the vaporization coefficient and $T_{v}$ is the prespecified temperature at which droplet evaporation is initiated (such as the liquid fuel's boiling temperature).

The governing equations are to be solved subject to the following boundary equations:

$$
\begin{aligned}
& u(x, 0)=v(x, 0)=0, u(x, \infty)=u_{\infty} \\
& T(x, 0)=T_{W}, T(x, \infty)=T_{\infty} \\
& \frac{\partial Y_{F}(x, 0)}{\partial x}=0, Y_{F}(x, \infty)=m_{F u} \\
& Y_{d}(x, \infty)=m_{d u}
\end{aligned}
$$

where the subscript "u" refers to conditions in the unburned mixture.

Eq. (1) is the continuity equation for the mixture. Its form follows from the implicit assumption that the liquid fuel volume fraction is sufficiently small. Therefore, the transport properties will be supposed to be determined primarily by the properties of the gaseous species. It is further assumed that the various transport coefficients such as thermal conductivity, diffusion coefficients, specific heat at constant temperature, latent heat of vaporization of the liquid droplets, etc., can be satisfactorily specified by representative constant values. It is assumed that the velocity of propagation of the flame is much less than the velocity of sound so that dynamic compressibility effects in the mixture can be neglected. Thus, the density becomes only a function of the temperature through the gas law. The droplets are taken to be in dynamic equilibrium with their host surroundings so that no momentum equations need to be solved for the droplets, and the appropriate drag-related term in the gas phase momentum Eq. (2) need not be considered. Eq. (3) is the energy conservation equation written in terms of temperature. Note the source/sink terms on the right hand side corresponding to (a) heat release by chemical reaction (second term) and (b) heat loss due to absorption of heat by droplets for vaporization (third term). Eq. (4) is the mass fraction conservation equation for fuel vapor with the last term on the right hand side the source term for the production of fuel vapor by evaporating droplets. In order to model the spray of liquid fuel droplets the sectional approach was exploited and a mono-sectional description is given here for simplicity at the current stage [16]. As mentioned, the droplets are viewed from a far-field vantage point, i.e. their average velocity is equal to that of their host environment. In addition, the temperature of the droplets is taken as equal to that of the surroundings; essentially, the droplets heat-up time is small compared with the characteristic time associated with their motion. Here, use of these latter two assumptions is made mainly for mathematical tractability but also in order to reduce some of the physical clutter to allow us to focus on the main mechanisms at play. Eq. (5) is the mass fraction conservation equation for the oxidiser. The use of the Heaviside function, $H$, in Eq. (8) implies that appreciable evaporation of the fuel droplets only commences when the local temperature reaches a prescribed value, $T_{v}$, say the liquid fuel's boiling temperature. The conditions under which the aforementioned spray-related assumptions (based on relevant scaling) are applicable are discussed at length in [17].

By applying appropriate transformations [15], assuming a unity Lewis number and then normalizing (see APPENDIX) this set of equations can be written in terms of a similarity variable, $\eta$, as follows:

$$
\begin{aligned}
& f^{\prime \prime \prime}+f f^{\prime \prime}+1-\left(f^{\prime}\right)^{2}=0 \\
& \theta^{\prime \prime}+f \theta^{\prime}=-\alpha \omega+\alpha L^{\prime} S_{v} \\
& y_{F}^{\prime \prime}+f y_{F}^{\prime}=\alpha \omega / \vartheta_{F}-\alpha S_{v} \\
& y_{O}^{\prime \prime}+f y_{O}^{\prime}=\alpha \omega / \vartheta_{O} \\
& f y_{d}^{\prime}=\alpha S_{v}
\end{aligned}
$$

where $f$ is related to the stream function which satisfies the continuity equation identically (for details see [12] and [15]), and

$$
\begin{aligned}
& \omega=\rho \alpha A m_{O u}\left(m_{F u}+m_{d u}\right) y_{F} y_{O} \exp \left(-\theta / \theta_{a}\right) \\
& S_{v}=\rho C y_{d} H\left(\eta_{v}-\eta\right) \\
& \alpha=(1 / a \rho)
\end{aligned}
$$


with $\eta_{v}$ being the location where the temperature at which the onset of evaporation occurs.

The boundary conditions become:

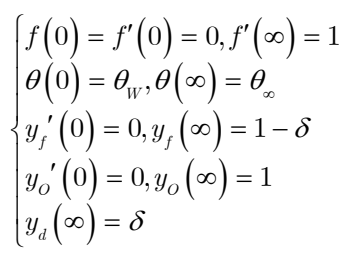

which must be supplemented with matching conditions of continuity on $\theta$ and $y_{f}$ and their first derivatives at $\eta_{v}$ where evaporation begins.

\section{Solution}

The solution for the stream function (Eq. (9)) is found numerically, and used when solving for the thermal and species fields. As far as the latter are concerned, in the limit of infinitely large activation energy chemical reaction will be negligible, but for large yet finite values chemical activity will be initiated close to the wall. The structure of the solution we seek should therefore be comprised of an outer "frozen" solution of a convective-diffusive nature merging with an inner solution near the wall which is dominated by a reactive-diffusive nature. We therefore determine the solutions in the outer region first taking the nonlinear chemical kinetic terms to be negligible. For ease, use is made of a new coordinate

$$
\xi=N(\eta) / N(\infty)
$$

with

$$
N(\eta) \equiv \int_{0}^{\eta} \exp \left(-\int_{0}^{\eta^{\prime}} f\left(\eta^{\prime \prime}\right) d \eta^{\prime \prime}\right) d \eta^{\prime}
$$

and the thermal field is found to be

$$
\theta_{\text {frozen }}(\xi)= \begin{cases}\frac{\left(\theta_{v}-\theta_{\infty}\right) \xi-\theta_{v}+\theta_{\infty} \xi_{v}}{\xi_{v}-1} & , 1 \geq \xi \geq \xi_{v} \\ \theta_{\text {Wall }}+\left[\theta_{v}-\theta_{\text {Wall }}-L^{\prime} \Delta \delta m\left(\xi_{v}\right)\right] \frac{\xi}{\xi_{v}}+L^{\prime} \Delta \delta m(\xi) & , \xi_{v} \geq \xi \geq 0\end{cases}
$$

The fuel vapor profile is given by

$$
y_{F, \text { frozen }}(\xi)=\left\{\begin{array}{lr}
1-\delta-\Delta \delta(\xi-1) m^{\prime}\left(\xi_{v}\right) & , 1 \geq \xi \geq \xi_{v} \\
1-\delta-\Delta \delta\left[\left(\xi_{v}-1\right) m^{\prime}\left(\xi_{v}\right)+m(\xi)-m\left(\xi_{v}\right)\right], & \xi_{v} \geq \xi \geq 0
\end{array}\right.
$$

The oxygen profile is simply

$$
y_{O, \text { frozen }}(\xi) \equiv 1
$$

and in Eqs. (19) and (20) use was made of the liquid fuel mass fraction profile

$y_{d}=\delta, \quad \eta \geq \eta_{v}$

$y_{d}=\delta \exp \left[\Delta\left(\Lambda(\eta)-\Lambda\left(\eta_{v}\right)\right)\right], \quad \eta_{v} \geq \eta \geq 0$

with

$$
\Lambda(\eta) \equiv \int \frac{d \eta}{f(\eta)}
$$

and the spray related evaporation function $m(\xi)$ which is the $\xi$-coordinate version of the following function written in terms of the similarity variable

$r(\eta)=\int_{0}^{\eta}\left[\exp \left(-\int_{0}^{\eta^{\prime}} f\left(\eta^{\prime \prime}\right) d \eta^{\prime \prime}\right)\left\{\int_{0}^{\eta^{\prime}} \exp \left[\Delta\left(\Lambda\left(\eta^{\prime \prime}\right)-\Lambda\left(\eta_{v}\right)\right)+\int_{0}^{\eta^{\prime \prime}} f\left(\eta^{\prime \prime}\right) d \eta^{\prime \prime}\right]\right\} d \eta^{\prime}\right.$

The location of the onset of evaporation is found from the implicit equation

$$
\frac{\left(\theta_{v}-\theta_{\infty}\right) N^{\prime}\left(\eta_{v}\right)}{N\left(\eta_{v}\right)-N(\infty)}=\frac{\left(\theta_{v}-\theta_{W}-L^{\prime} \Delta \delta r\left(\eta_{v}\right)\right) N^{\prime}\left(\eta_{v}\right)}{N\left(\eta_{v}\right)}+L^{\prime} \Delta \delta r^{\prime}\left(\eta_{v}\right)
$$


Armed with the outer frozen solution, we are in a position to develop the inner solution close to the stagnation point at which chemical reaction will occur. Asymptotic methods are tailor-made for this purpose once a small parameter has been identified. Here we choose to use

$\varepsilon=\frac{\theta_{W}^{2}}{\theta_{a}} ; \theta_{a}=\frac{E c_{p}}{R q} ; \theta_{W}=\frac{T_{W} c_{p}}{q} ; \beta=\theta_{W}-\theta_{v}$

The temperature distribution in the outer region has the following form

$\theta_{\text {outer }}=\theta_{v}+A_{0}\left(\xi_{v}-\xi\right)+\varepsilon A_{1}\left(\xi_{v}-\xi\right)+O\left(\varepsilon^{2}\right)$

where the constants $A_{0}, A_{1}$ will be found by matching the inner and outer solutions.

In order to continue the analysis we introduce a stretched inner coordinate

$\chi=\beta \xi / \varepsilon$

Now, the solution in the inner region will be the frozen outer solution with the addition of a perturbation due to chemical reaction, viz.

$\theta_{\text {inner }}(\chi)=\theta_{\text {frozen }}(\chi)+\varepsilon \phi(\chi)$

After much algebraic manipulation it can be shown that the function $\phi$ must satisfy

$\frac{d^{2} \phi}{d \chi^{2}}=-\frac{\sigma}{2} \exp \left(\phi-\frac{\chi}{\xi_{v}}\left(1+\frac{L^{\prime} \Delta \delta m\left(\xi_{v}\right)}{\beta}\right)-\frac{\theta_{a} L^{\prime} \Delta \delta m\left(\frac{\varepsilon \chi}{\beta}\right)}{\theta_{W}^{2}}\right)$

where

$\sigma \equiv \frac{2 \mu \varepsilon N(\infty)^{2}}{\beta^{2}} \cdot\left(1-\left.\frac{\xi_{v}}{\vartheta_{O}} \beta \frac{d \phi}{d \chi}\right|_{0}\right)\left(\begin{array}{l}1-\delta-\Delta \delta\left[\left(\xi_{v}-1\right) m^{\prime}\left(\xi_{v}\right)-m\left(\xi_{v}\right)\right] \\ -\left.\frac{\xi_{v}}{\vartheta_{F}} \beta \frac{d \phi}{d \chi}\right|_{0}\end{array}\right) \exp \left(-\frac{\theta_{a}}{\theta_{W}}\right)$

with $\sigma$ being a suitably defined Damkohler number. When $\sigma \rightarrow 0$ chemical reaction will be negligible, whereas when $\sigma \rightarrow \infty$ it is very fast. Eq. (30) is subject to the boundary condition $\phi(\chi=0)=0$ which stems from the $O(1)$ nature of the temperature at the stagnation point. The second boundary condition is obtained by matching the inner and outer solutions and is given by:

$\left.\frac{\partial \phi}{\partial \chi}\right|_{\chi \rightarrow \infty}=\frac{L^{\prime} \Delta \delta m\left(\xi_{v}\right)}{\xi_{v} \beta}$

The solution of Eq.(30) is

$\phi^{*}(\tau)=\tau+\ln \left[c_{1}\left(1-\tanh ^{2}\left(\frac{\sqrt{c_{1}\left(c_{2}+\tau\right)^{2}}}{2}\right)\right) / \sigma^{*}\right]$

where

$\tau=\frac{\chi}{\xi_{v}}\left(1+\frac{L^{\prime} \Delta \delta m\left(\xi_{v}\right)}{\beta}\right)$

$\phi^{*}=\phi-\frac{\theta_{a} L^{\prime} \Delta \delta m\left(\frac{\varepsilon \chi}{\beta}\right)}{\theta_{W}^{2}}$

$\sigma^{*}=\frac{\sigma}{\left[\frac{1}{\xi_{v}}\left(1+\frac{L^{\prime} \Delta \delta m\left(\xi_{v}\right)}{\beta}\right)\right]^{2}}$

and where $c_{1}, c_{2}$ are constants to be found from the boundary conditions. The condition at the wall leads to:

$\phi^{*}(0)=\ln \left(\frac{c_{1}}{\sigma^{*}}\right)+\ln \left[1-\tanh ^{2}\left(\frac{\sqrt{c_{1} c_{2}^{2}}}{2}\right)\right]=0$ 
whereas the outer condition yields

$$
c_{1}=\left(1-\frac{L^{\prime} \Delta \delta m\left(\xi_{v}\right)}{\beta+L^{\prime} \Delta \delta m\left(\xi_{v}\right)}\right)^{2}
$$

By substituting back into Eq.(38), after some algebra it is found that

$$
\left|c_{2}\right|=\ln \left[\frac{1 \pm \sqrt{1-\frac{\sigma^{*}}{c_{1}}}}{1 \sqrt{1-\frac{\sigma^{*}}{c_{1}}}}\right] / \sqrt{c_{1}}
$$

from which it can deduced that in order to guarantee a solution the condition $\sigma^{*} \leq c_{1}$ must be satisfied. This implies that $\sigma^{*}=c_{1}$ is a transition point between a burning and non-burning solution or, putting it another way, between a situation in which ignition occurs or does not. In full, the condition reads

$\sigma=1 / \xi_{v}^{2}$

where $\sigma$ is defined in Eq. (31). The role that the spray plays in this ignition criterion is clearly represented by the heat loss terms resulting from the heat absorbed by the droplets for evaporation, by the production of fuel vapor that exhibits itself via the second bracketed term on the RHS of Eq. (31) and by the location of the onset of evaporation $\xi_{v}$. It is of interest to note that if no spray is present, i.e. $\delta=0$, a single phase gas situation is under consideration, the criterion Eq. (40) collapses to that of [12].

\section{Results and discussion}

Use was made of the analytical solution in the previous section to examine the effect of heat loss and fuel spray parameters on conditions for spray flame propagation and extinction. The data used for the calculations was as follows (unless otherwise specified): $Q=1.279 \times 10^{7} \mathrm{~J} / \mathrm{kg}, L=0.04 Q, \lambda=0.02512 \mathrm{Wm} / \mathrm{K}, \mathrm{A}=10^{10} \mathrm{~s}^{-1}$, $E=2 \times 10^{8} \mathrm{~J} / \mathrm{kmol}, \quad c_{p}=1255.92 \mathrm{~J} / \mathrm{kgK}, T_{u}=300 \mathrm{~K}, T_{v}=400 \mathrm{~K}, \quad a=1000 \mathrm{sec}^{-1}$. The chemical kinetic scheme employed concerns the burning of $n$-decane and relevant thermochemical data was taken from [18] and [19]. By specifying the initial fraction of liquid fuel in the total fuel (vapor +liquid) in the fresh mixture, $\delta$, it can be shown that the mass fractions in the fresh mixture are given by the following expressions:

$\left(m_{O u}, m_{d u}, m_{F u}\right)=\left(s\left\{1-m_{d u}-m_{F u}\right\}, \frac{s \delta}{\left(1+\alpha_{O F} / \phi\right)}, m_{d u} \frac{(1-\delta)}{\delta}\right)$

unless $\delta=0$ for which

$m_{F u}=\frac{s}{\left(s+\alpha_{O F} / \phi\right)}$

where $s$ is the mole fraction of oxygen in the fresh mixture, $\alpha_{O F}$ is the stoichiometric coefficient and $\phi$ is the equivalence ratio. Here $\phi$ is taken as $5, \alpha_{O F}=3.5$ and $s=1$.

It is clear that, given a set of data relating to the gas and liquid phases, condition (40) and Eq. (31) provide a relationship from which the critical value of the stagnation point temperature can be extracted. To examine the influence of the spray-related parameters on this temperature these parameters were varied whereas the gasrelated parameters were held fixed. Fig. 2 is an example in which contours of selected critical temperatures are drawn in the $C-\delta$ plane, where it will be recalled that $C$ is the evaporation coefficient and $\delta$ is the fraction of liquid fuel in the initial vapor+liquid fuel mass fraction. Consider, for example, the contour of $T_{W}=1570 \mathrm{~K}$. Points (i.e. liquid phase conditions to the right of this contour) will not permit ignition, whereas points to the left will support the existence of a flame in the vicinity of the stagnation point. Moving to the right of any contour for a fixed value of $C$ implies that the initial liquid load is greater, thereby effectively increasing the heat loss to the system required to evaporate the droplets. Similarly, for a fixed value of $\delta$ increasing the evaporation coefficient produces more focusing of the heat loss borne by the system. In both cases a greater value of $T_{W}$ would therefore be required to initiate chemical reaction.

Another way to view this behaviour is illustrated in Fig. 3 where the actual critical temperature for ignition is plotted as a function of the evaporation coefficient for different initial liquid loads. Also, appearing is the horizontal curve for a pure gaseous fuel, for the sake of comparison. The Influence of both the spray parameters is rather clear. Take, for example, $C=600$. The presence of only liquid fuel instead of purely gaseous fuel produces a $4 \%$ increase in the critical temperature for ignition. 


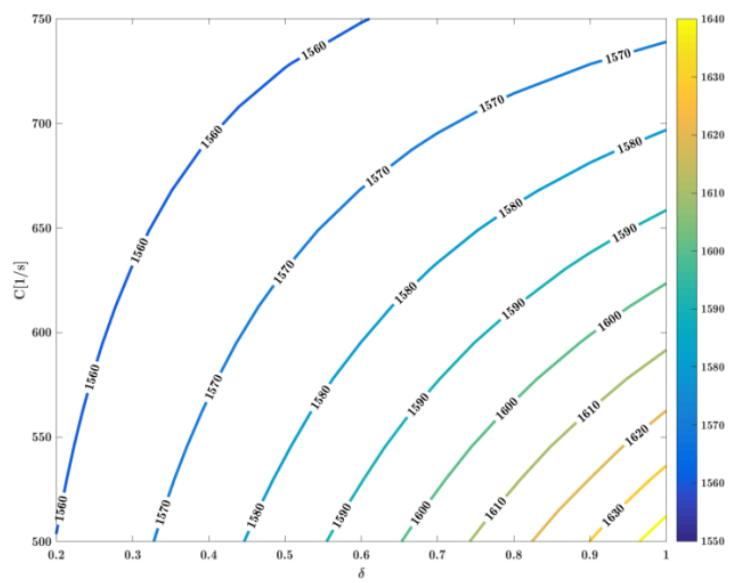

Figure 2: Influence of vaporization coefficient and total initial liquid load on conditions for ignition in stagnation point flow; points to the left of a given temperature contour permit ignition.

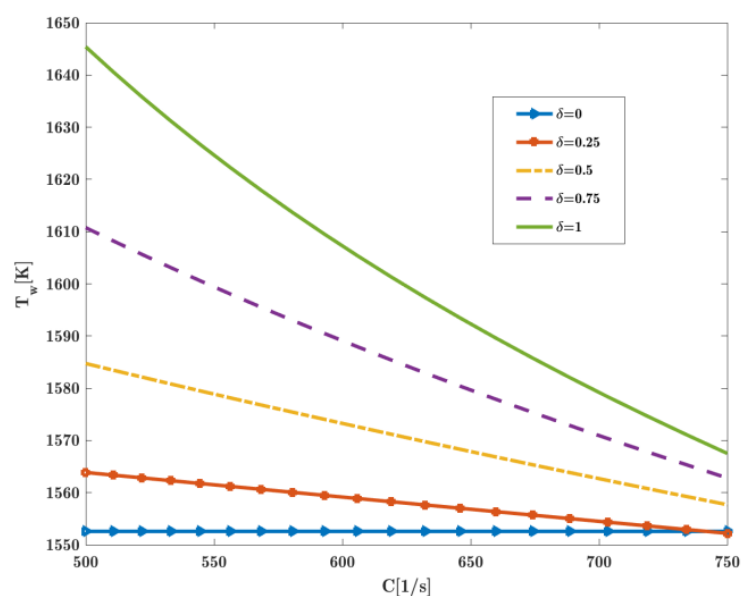

Figure 3: Influence of vaporization coefficient on critical stagnation-point temperature for ignition, for various total initial liquid loads, in stagnation point flow.

Finally, in Fig. 4 we show the influence of the vaporization coefficient on the mass fraction of fuel vapor at the stagnation point. This term is the second bracketed term on the right hand side of Eq. (31) before the exponential term, and represents the role of the fuel vapor produced by droplet evaporation in the chemical reaction.

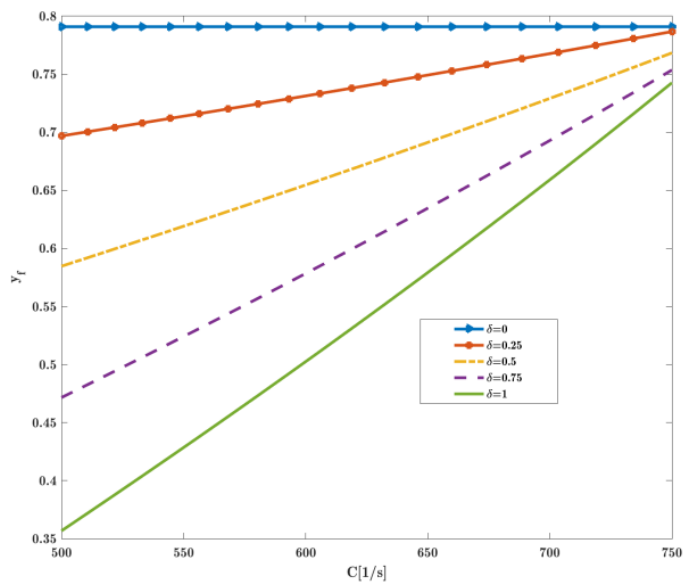

Figure 4: Influence of vaporization coefficient on stagnation-point mass fraction of fuel vapor at ignition, for various total initial liquid loads, in stagnation point flow. 
It can be observed that the higher the initial liquid load the lower the value of fuel vapor mass fraction. As a consequence, to compensate a higher temperature must be supplied at the stagnation point for ignition to occur. This is readily seen to be the case by referring to Fig. 3 .

\section{Conclusions}

A new steady-state/static analysis of conditions for ignition of an air-fuel spray mixture in stagnation point flow impinging on an isothermal wall was presented. The analysis leads to a criterion for ignition that includes effects of the flow field, the reactants and the fuel spray-related parameters. Numerical calculations using the formula mapped the way in which the latter impact on whether ignition will occur or not.

The analysis is predicated on a number of simplifying assumptions which, nevertheless, enable a first insight into the complexities of spray ignition to be uncovered. Further research into relaxing some of these assumptions without having to resort to a complete CFD code to obtain a solution are currently ongoing and will be reported in the future. Experimental evidence to validate or suggest modification of the theory will be welcomed by the authors.

\section{Acknowledgements}

JBG thanks the Lady Davis Chair in Aerospace Engineering for partial support of this research.

\section{Appendix}

In this Appendix the normalized quantities appearing in the governing equations are listed:

$$
\left\{\begin{array}{l}
\theta=\frac{c_{p} T}{q} ; \theta_{a}=\frac{E c_{p}}{R q} ; L^{\prime}=\frac{L\left(m_{d u}+m_{f u}\right)}{q} ; y_{f}=\frac{Y_{f}}{m_{d u}+m_{f u}} ; y_{d}=\frac{Y_{d}}{m_{d u}+m_{f u}} ; y_{O}=\frac{Y_{O}}{m_{O u}} ; \delta=\frac{m_{d u}}{m_{d u}+m_{f u}} ; \\
\Delta=\frac{C}{a} ; \vartheta_{O}=m_{O u} ; \vartheta_{F}=m_{d u}+m_{f u} ; \mu=\frac{A m_{O u}\left(m_{F u}+m_{d u}\right)}{a} ; \\
s=\int_{0}^{x} \rho_{0}\left(x^{\prime}\right) \mu_{0}\left(x^{\prime}\right) u_{0}\left(x^{\prime}\right) d x^{\prime} ; \eta=\frac{u_{0}}{\sqrt{2 s}} \int_{0}^{y} \rho\left(x, y^{\prime}\right) d y^{\prime}
\end{array}\right.
$$

In these definitions $q$ is the heat of reaction.

\section{References}

[1] Neophytou, A., "Spark ignition and flame propagation", PhD dissertation, University of Cambridge, 2010.

[2] Aggarwal, S.K., 2014, Progress in Energy and Combustion Science, 45, pp. 79-107.

[3] Kikby, L.L. and Schmitz, R.A., 1966, Combustion and Flame, 10, pp. 205-219.

[4] Linan, A., 1974, Acta Astronautica, 1, pp. 1007-1039.

[5] Matalon, M. and Ludford, G.S.S., 1979, Acta Astronautica, 6, pp. 1377-1386.

[6] Aggarwal, S.K. and Sirignano, W.A., 1984, Proceedings of the Combustion Institute, 20, pp. 1773-1780.

[7] Aggarwal, S.K. and Sirignano, W.A., 1986, Combustion Science and Technology, 46(3-6), pp. 289-300.

[8] Aggarwal, S.K. and Sirignano, W.A., 1988, AIAA Journal of Propulsion, 4(1), pp. 14-21.

[9] Kats, G. and Greenberg, J.B., Scitech 2016, AIAA Paper 2016-1029, San Diego, USA, 4-8 January 2016.

[10] Kats, G. and Greenberg, J.B., ILASS - Europe 2016, 27th Annual Conference on Liquid Atomization and Spray Systems, 4-7 September 2016, Brighton, UK.

[11] Neophytou, A., Mastorakos, E. and Cant, R.S., 2011, Proceedings of the Combustion Institute, 33, pp. 21352142.

[12] Law, C.K., 1978, International Journal of Heat and Mass Transfer, 21, pp. 1363-1368.

[13] Chen, Z.H., Lin, T.H. and Sohrab, S.H., 1988, Combustion Science and Technology, 60(1-3), pp. 63-77.

[14] Hsu, A-K. and Lin, T.H., 2003, Combustion Science and Technology, 175(12), pp. 2141-2159.

[15] Chung, P.M., Chemically reacting non-equilibrium boundary layers, in Advances in Heat Transfer, Academic press, NY, 1965.

[16] Greenberg, J.B., Silverman, I. and Tambour, Y., 1993, Combustion and Flame, 93, pp. 90-96.

[17] Sanchez, A.L, Urzay, J. and Linan, A., 2015, Proceedings of the Combustion Institute, 35, pp. 1549-1577.

[18] Stull, D.R. and Prophet, H., JANAF Thermochemcial Tables (2nd ed.), NSRDS-NBS 37., Washington, DC 20234, 1971.

[19] Vargaftik, N.B., Tables on the Thermophysical Properties of Liquids and Gases (2nd ed.), John Wiley and Sons, 1975. 